\title{
SHARP OFF-DIAGONAL WEIGHTED WEAK TYPE ESTIMATES FOR SPARSE OPERATORS
}

\author{
QIANJUN HE AND DUNYAN YAN
}

Abstract. We prove sharp weak type weighted estimates for a class of sparse operators that includes majorants of standard singular integrals, fractional integral operators, and square functions. These bounds are known to be sharp in many cases, and our main new result is the optimal bound

$$
[w, \sigma]_{A_{p, q}^{\alpha}}^{\frac{1}{q}}\left[w^{q}\right]_{A_{\infty}}^{\frac{1}{v}-\frac{1}{p}} \lesssim[w]_{A_{p, q}}^{\frac{1}{q}}[w]_{A_{p, q}}^{\frac{1}{v}-\frac{1}{p}}=[w]_{A_{p, q}}^{\frac{1}{v}-\frac{\alpha}{d}}
$$

for $p>v$ and Sobolev type condition $\frac{1}{q}+\frac{\alpha}{d}=\frac{1}{p}$. For $v \leqslant q \leqslant \frac{v}{1-\frac{v \alpha}{d}}$, we also obtain the bounds $[w]_{A_{p, q}}^{\frac{1}{q}}$ and it has an additional logarithmic factor, taking the form $\left(1+\log \left[w^{q}\right]_{A_{\infty}}\right)^{\frac{1}{v}}$. Moreover, we study a class of sparse maximal operators and give the weak type off-diagonal two-weight sharp bound.

Mathematics subject classification (2010): 42B20, 42B25.

Keywords and phrases: $A_{p, q}^{\alpha}-A_{\infty}$ estimates, off-diagonal estimates, sparse operators, square functions.

\section{REFERENCES}

[1] F. Bernicot, D. Frey, And S. Petermichl, Sharp weighted norm estimates beyond CalderónZygmund theory, Anal. \& PDE, 9, 5 (2016), 1079-1113.

[2] C. Cascante, J. M. Ortega And E. Verbitsky, Nonlinear potentials and two weight trace inequalities for general dyadic and radial kernels, Indiana Univ. Math. J., 53, 3 (2004), 845-882.

[3] J. COnde-Alonso And G. REY, A pointwise estimate for positive dyadic shifts and some applications, Math. Ann., 365, (2016), 1111-1135.

[4] D. CRUZ-URIBE AND K. MoEn, A fractional Muckenhoupt-Wheeden theorem and its consequences, Integr. Equat. Oper. Th., 76, 3 (2013), 421-446.

[5] D. CRUZ-URIBE AND K. MoEn, One and two weight norm inequalities for Riesz potentials, Illinois J. Math., 57, 1 (2013), 295-323.

[6] S. FACKLER AND T. P. HytÖNEN, Off-diagonal sharp two-weight estimates for sparse operators, New York J. Math., 24, (2018), 21-42.

[7] C. Domingo-SAlazR, M. T. LACEY AND G. REY, Borderline weak type estimates for singular integrals and square functions, Bull. Lond. Math. Soc., 48, 1 (2016), 63-73.

[8] J. DuoAndikoetxeA, Extrapolation of weights revisited: new proofs and sharp bounds, J. Funct. Anal., 260, 6 (2011), 1886-1901.

[9] J. GARCÍA AND J. M. MARTELL, Two-weight norm inequalities for maximal operators and fractional integrals on non-homogeneous spaces, Indiana Univ. Math. J., 50, 3 (2001), 1241-1280.

[10] T. S. HÄNNINEN AND E. LoRIST, Sparse domination for the lattice Hardy-Littlewood maximal operator, Proc. Amer. Math. Soc., 147, (2019), 271-284.

[11] T. Hytönen, The sharp weighted bound for general Calderón-Zygmund operators, Ann. of Math. (2), 175, 3 (2012), 1473-1506.

[12] T. HYTÖNEN AND K. W. LI, Weak and strong $A_{p}-A_{\infty}$ estimates for square functions and related operators, Proc. Amer. Math. Soc., 146, (2018), 2497-2507.

[13] T. Hytönen And C. PÉREZ, Sharp weighted bounds involving $A_{\infty}$, Anal. \& PDE, 6, 4 (2013), 777-818. 
[14] M. T. LACEY AND K. W. LI, On $A_{p}-A_{\infty}$ type estimates for square functions, Math. Z., 284, 3-4 (2016), 1211-1222.

[15] M. T. Lacey, K. Moen, C. Pérez And R. H. Torres, Sharp weighted bounds for fractional integral operators, J. Funct. Anal., 259, 5 (2010), 1073-1097.

[16] M. T. LACEY, E. SAWYER, AND I. URIARTE-TUERO, Two weight inequalities for discrete positive operators, available at http://arxiv.org/abs/0911.3437.

[17] M. T. LACEY AND J. SCURRY, Weighted weak type estimates for square functions, availble at http://arxiv.org/abs/1211.4219.

[18] J. LAI, A new two weight estimates for a vector-valued positive operator, available at http://arxiv.org/abs/1503.06778.

[19] A. K. LERNER, Sharp weighted norm inequalities for littlewood-Paley operators and singular integrals, Adv. Math., 226: 3912-3926, 2011.

[20] A. K. LeRner, Mixed $A_{p}-A_{r}$ inequalities for classical singular integrals and Littlewood-Paley operators, J. Geom. Anal., 23, 3 (2013), 1343-1354.

[21] A. K. LERnER, On pointwise estimates involving sparse operators, New York J. Math., 22, (2016), 341-349.

[22] A. K. Lerner AND F. NAZARov, Intuitive dyadic calculus: the basics, Expo. Math., 37, 3 (2019), 225-265.

[23] B. Muckenhoupt And R. L. WheEden, Weight norm inequalities for fractional integrals, Trans. Amer. Marh. Soc., 192, (1974), 2611-274.

[24] B. Muckenhoupt, Weighted norm inequalities for the Hardy-Littlewood maximal function, Trans. Amer. Math. Soc., 165, (1972), 207-226.

[25] M. C. Pereyra, Dyadic harmonic analysis and weighted inequalities: the sparse revolution, New Trends in Applied Harmonic Analysis, Volume 2, 159-239, 2019.

[26] P. Zorin-KRANICH, $A_{p}-A_{\infty}$ estimates for multilinear maxinal and sparse operators, JAMA, 138, (2019), 871-889. 